\title{
Cancelling Prism Adaptation by a Shift of Background: A Novel Utility of Allocentric Coordinates for Extracting Motor Errors
}

\author{
Motoaki Uchimura ${ }^{1,2,4}$ and Shigeru Kitazawa $\mathbf{a}^{1,2,3,4}$ \\ ${ }^{1}$ Dynamic Brain Network Laboratory, Graduate School of Frontier Biosciences, and 2Department of Brain Physiology, Graduate School of Medicine, Osaka \\ University, Suita, Osaka 565- 0871, Japan, ${ }^{3}$ Center for Information and Neural Networks, National Institute of Information and Communications \\ Technology and Osaka University, Suita, Osaka 565-0871, Japan, and ${ }^{4}$ Department of Neurophysiology, Graduate School of Medicine, Juntendo University, \\ Bunkyo, Tokyo 113-8421, Japan
}

Many previous studies have reported that our brains are able to encode a target position not only in body-centered coordinates but also in terms of landmarks in the background. The importance of such allocentric memory increases when we are forced to complete a delayed reaching task after the target has disappeared. However, the merit of allocentric memory in natural situations in which we are free to make an immediate reach toward a target has remained elusive. We hypothesized that allocentric memory is essential even in an immediate reach for dissociating between error attributable to the motor system and error attributable to target motion. We show here in humans that prism adaptation, that is, adaptation of reaching movements in response to errors attributable to displacement of the visual field, can be cancelled or enhanced simply by moving the background in mid-flight of the reaching movement. The results provide direct evidence for the novel contribution of allocentric memory in providing information on "where I intended to go," thereby discriminating the effect of target motion from the error resulting from the issued motor control signals.

\section{Introduction}

Our brains are able to memorize target positions not only in body-centered coordinates but also in terms of landmarks, such as a square (Fig. 1a), in the background (McIntyre et al., 1998; Burgess et al., 2004; Krigolson and Heath, 2004; Lemay et al., 2004; Sheth and Shimojo, 2004; Obhi and Goodale, 2005; Krigolson et al., 2007; Byrne and Crawford, 2010; Byrne et al., 2010; Chen et al., 2011). These studies have often adopted delayed reaching tasks in which participants reach for a target that disappeared a moment ago. The errors generally decreased when landmarks were available compared with when they were unavailable. However, the effect of the landmarks was much reduced when subjects made an immediate reach and became obvious only when the delay was as long as $\sim 2$ s or more (Carrozzo et al., 2002; Sheth and Shimojo, 2004; Obhi and Goodale, 2005). Admitting that the allocentric coordinate is useful in memorizing "where to go" (Fig. 1a, left), there are few natural situations that involve

Received Dec. 13, 2012; revised March 4, 2013; accepted March 20, 2013.

Author contributions: M.U. and S.K. designed research; M.U. and S.K. performed research; M.U. and S.K. analyzed data; M.U. and S.K. wrote the paper.

The study was partly supported by Human Frontier Science Program Research Grant RGP0049/2009-C and a Health Labour Sciences Research Grant from the Ministry of Health Labour and Welfare (S.K.).

The authors declare no competing financial interests.

Correspondence should be addressed to Shigeru Kitazawa, Dynamic Brain Network Laboratory, Graduate

School of Frontier Biosciences, Osaka University, 1-3 Yamadaoka, Suita, Osaka 565-0871, Japan. E-mail: kitazawa@fbs.osaka-u.ac.jp.

DOI:10.1523/JNEUROSCI.5702-12.2013

Copyright (C) 2013 the authors $\quad 0270-6474 / 13 / 337595-08 \$ 15.00 / 0$ reaching for a target that disappeared $>2 \mathrm{~s}$ ago. Thus, the realistic utility of the allocentric memory is yet to be discovered.

In natural situations, a target often moves (Fig. $1 a$, right). However, we are never able to compensate for a target motion that occurred within the last $\sim 150 \mathrm{~ms}$ of a reaching movement (Day and Lyon, 2000). Thus, the apparent discrepancy between the target and the hand (apparent error) consists of two components: one attributable to the error in the motor system (here termed "motor error") and another attributable to the target motion. For improving motor skill, it is essential to estimate the net motor error by discounting the error attributable to target motion. We here hypothesize that the allocentric memory is essential for dissociating error attributable to the motor system from error attributable to target motion by encoding "where I intended to go" (Fig. 1 $a$, right).

To test this hypothesis, we introduced a large apparent error using a lateral displacing prism and presented a frame in the background (Fig. 1b). In a control condition, the frame remained still (no shift): the apparent error itself was expected to serve as the motor error to be corrected, and the error decreased trial by trial as in the traditional prism adaptation paradigm. In another condition, the frame was displaced in the direction of prism displacement in midflight of reaching (ipsilateral shift), with no movement of the target itself. In this condition, the original target position decoded in the frame coordinate would shift with the frame (dotted circle), and the hand would be likely to land on the decoded position. The motor error would then be judged as much smaller than the apparent error, and prism adaptation would be expected to be reduced. In a third condition, the frame was displaced in the opposite direction (con- 
tralateral shift). In this case, prism adaptation was likely to be enhanced. We show in the following that the prism adaptation was modulated as predicted.

\section{Materials and Methods}

Participants. Thirty-seven young adults (33 men and four women, aged 19-28 years) with normal vision or vision that was corrected to normal with contact lenses participated in the study. Participation was discontinued for seven participants because of low success rates during a training session in terms of reaction time and movement duration. All participants were naive to the purpose of the experiments, and none reported neurological history. All participants were right-handed (laterality quotient, +80 to +100 ) according to the Edinburgh Inventory (Oldfield, 1971). The study received approval from the institutional ethics committee, and all participants gave informed written consent before the experiments.

Apparatus and general task procedures. The participants were seated facing a tangent 22inch LCD screen $360 \mathrm{~mm}$ from their eyes with their heads restrained by a chin rest and a head band; they viewed the screen through a refractor (with two pairs of motor-driven wedge prisms, one for each eye) that restricted their view of the screen within the visual field to $\sim 30^{\circ}$ (Fig. 2a). Liquid crystal shutters were placed between the prisms and the eyes of the participants. The refractor was designed to achieve a desired displacement of $0-20$ diopter $\left(0-11.3^{\circ}\right)$ in any direction by rotation of the prisms with a command from a personal computer (Interface Corporation). The shutters were opened at the initiation of each trial when the participants pressed a button with their index fingers. The button was positioned 300 $\mathrm{mm}$ below and $100 \mathrm{~mm}$ ahead of the subject's eyes in the midsagittal plane. A target (a red cross, $15 \mathrm{~mm}=2.4^{\circ}$ wide) and background figures drawn with blue lines then appeared on a white background after a random delay (900-1200 ms). The position of the background figures was randomized from one trial to another so that the centers of the figures fell within a circular zone $\left(20 \mathrm{~mm}\right.$ radius, $\left.3.2^{\circ}\right)$ in the center of the visual field. A target was presented at a random location within a target zone $\left(20 \mathrm{~mm}\right.$ radius, $3.2^{\circ}$ ) placed in the center of the background figures. The participants were required to release the button within $300 \mathrm{~ms}$ (but after $150 \mathrm{~ms}$ ) from the appearance of the target and to touch the screen within $300 \mathrm{~ms}$ of the release. The shutters were closed at the release to block the vision of the hand and the target during the reach and were opened again at the touch, which was detected with a touch sensor (Touch Panel Laboratories), to allow the participants to see the target and the final position of the hand for $300 \mathrm{~ms}$. The participants were required to hold the final position of the hand for $1000 \mathrm{~ms}$ after the touch and were allowed to return the hand to the starting position when they heard a beep. The prisms were rotated for $500 \mathrm{~ms}$ during each intertrial interval even in blocks with no visual displacement so that the participants would not be able to infer the size of visual displacement from the sound of the motors. It took 5-6 s to complete one trial. When the reaction time or the movement time was outside the required range (failure trial), the shutters were kept closed at the touch with a low beep sound.
"Where I intended to go" in immediate reach in delayed reach

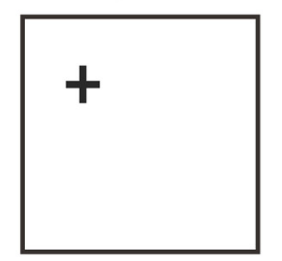

Blank ( 2 s)

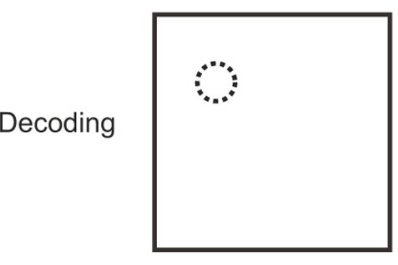

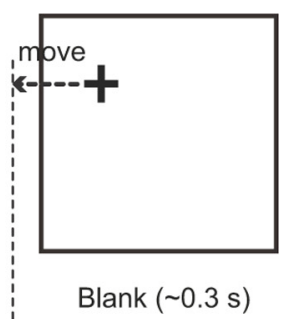

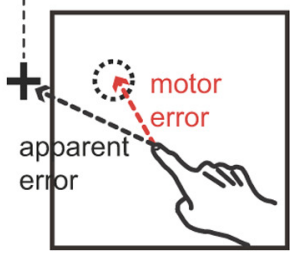

Figure 1. A novel utility of the allocentric memory of a target position. $\boldsymbol{a}$, Allocentric memory in terms of the background $2 \mathrm{~s}$ (left). We propose a novel use of the allocentric memory in an immediate reach (right). We hypothesize that the (n) motor system (red arrow) from error attributable error (black arrow) was introduced using a lateral displacing prism. The frame remained still (left) or was displaced in the direction of prism displacement (ipsilateral shift, middle) or in the opposite direction (contralateral shift, right) during a reaching movement. Assuming that our hypothesis is correct, the original target position decoded in terms of the frame condition and overestimated in the contralateral condition.

One session consisted of three periods of 30 trials. In the first and the third periods (pretest and posttest periods), the participant performed the reaching task without visual displacement or any shift of background figures. In the second period (exposure period), the visual field was displaced to the right or to the left by $20 \mathrm{~mm}\left(3.2^{\circ}\right)$, and the background figures were shifted by $20 \mathrm{~mm}$ in the same (ipsilateral shift condition) or the contralateral direction (contralateral shift condition) while the participant's vision was blocked with shutters. The size of visual field was restricted within $\sim 30^{\circ}$ so that objects in the background other than the background figures, such as the edges of the LCD screen, cannot be seen by the participants. The directions of visual displacement and the background figures were kept constant through the 30 trials in each exposure period. Failure trials were discarded, and each period was continued until the participants completed 30 successful trials. It took $\sim 8-10 \mathrm{~min}$ to complete one session.

The participants were instructed to ignore the background figures and to reach for the target as rapidly and precisely as possible without any conscious corrections. The participants were familiarized with the task 


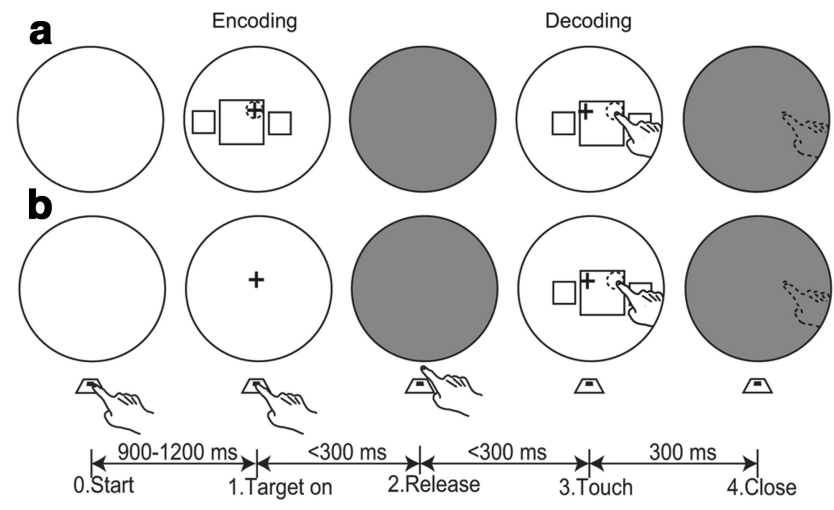

Figure 2. Task procedures. Each column schematically shows the status of the visual field (oval shape) and the position of the hand relative to the button and the screen. The shutters opened at 0 (Start) when the participant pressed the button. A target and background figures appeared after a random delay (900-1200 ms) at 1. Vision was blocked from 2 (Release) to 3 (Touch) and allowed again for $300 \mathrm{~ms}$. The background figures were presented both before (encoding) and after (decoding) the reach in experiments 1-3 $(\boldsymbol{a})$; they were presented only after the reach in experiment $4(\boldsymbol{b})$.

procedure beforehand until they had little difficulty in generating the task movement within the required time period. Each participant sat for one or two sessions per day. When there were two sessions, the participants rested for 10-20 min between sessions. Thirty trials without visual displacement were added to ensure washout of any aftereffects before and after each session.

Experiment 1: effects of ipsilateral and contralateral shifts of the background. Six participants completed this experiment. Triple squares, a center square $\left(80 \mathrm{~mm}, 13^{\circ}\right)$ between two smaller squares $\left(40 \mathrm{~mm}, 6.3^{\circ}\right)$, were presented as a background both before (encoding period) and after (decoding period) the blank (Fig. 2a). There were two $\times$ three conditions depending on the direction of visual displacement (left or right) and the direction of the background shift (ipsilateral, contralateral, and no background shift conditions; Fig. 1b). Each participant completed six sessions, one for each of the two $\times$ three conditions. The order of the conditions was counterbalanced across the participants.

Experiment 2: effects of the size of background (see Fig. 4). Six participants completed this experiment. The size of background (triple squares) was altered in five steps with the background always shifted in the ipsilateral direction. There were two $\times$ five conditions: two directions of visual displacement (left or right) by five sizes of background $(0,8,40,80$, and $120 \mathrm{~mm}$ width of the center square; $0,1.3,6.3,13,18^{\circ}$ width; see Fig. $4 a$ ). Each participant completed 10 sessions, one for each of the two $\times$ five conditions. The orders of conditions were counterbalanced within and across the participants.

Experiment 3: effects of the complexity of background (see Fig. 5). Twelve participants completed this experiment. The complexity of the background was altered in six steps (no background, single line, parallel lines, single square, triple squares, and multiple squares; see Fig. $5 a$ ), with the background always shifted in the ipsilateral direction. The length of the line and the width of the center square were fixed at $80 \mathrm{~mm}$. For the single line condition, one of the four sides of the center square was assigned for each participant (three participants for each side). For the parallel line condition, one of two pairs (vertical or horizontal pairs) was assigned for each participant (six participants for each). For each participant, the direction of visual displacement was fixed either to the right (six participants) or to the left (six participants). Each participant completed six sessions, one for each complexity. The order of the conditions was counterbalanced across the participants.

Experiment 4: necessity of the background during the encoding phase. Six participants completed this experiment. The triple-square background (center square, $40 \mathrm{~mm}, 6.3^{\circ}$ in width) was presented in three ways: (1) the background was not presented (no background condition); (2) the background was presented both before and after the blank period with an ipsilateral shift (ipsilateral shift condition); and (3) the background was presented only after the blank (post-presentation condition). Each participant sat for the experiment in each of the six conditions (2 directions of prisms $\times 3$ background conditions). The order of the conditions was counterbalanced across the participants.

Data analyses. The horizontal errors in success trials (30 trials for each period) were analyzed. The mean horizontal error during the pretest period was regarded as a bias and was subtracted from the horizontal errors obtained during the exposure and posttest periods. The bias-free horizontal errors were then used for estimating the asymptote in the exposure period and the size of the aftereffect in the posttest period using a discrete model formulated as follows:

$$
h(n)=h(n-1)-k(\tilde{h}(n-1)-\alpha),
$$

where $h(n), \tilde{h}(n), k$, and $\alpha$ denote, respectively, the estimated horizontal error in the $n$th trial, the observed horizontal error in the $n$th trial, a constant rate at which the error is assumed to decrease, and an asymptote to which the error is assumed to converge. The model defines a learning algorithm in which the error decreases by an amount proportional to the error observed in the preceding trial to an asymptote. Except for the introduction of the asymptote, the model was the same as that used in our previous studies (Kitazawa et al., 1995; Kitazawa and Yin, 2002). The MATLAB optimization toolbox was used for least-squares estimation of $h(1), k$, and $\alpha$. The estimated asymptote $(\alpha)$ during the exposure period and the estimated initial error, $h(1)$, during the posttest period (aftereffect) were further compared across conditions using one-way repeatedmeasures ANOVA and a post hoc analysis (Ryan's method; Day and Quinn, 1989).

\section{Results \\ Effects of ipsilateral and contralateral shifts of the background (experiment 1)}

The results we obtained generally agreed with the predictions from our hypothesis. In the ipsilateral shift condition, the prism adaptation of a participant was almost completely cancelled (Fig. $3 a)$; the error during the exposure period persisted at $\sim 20 \mathrm{~mm}$ $\left(3.2^{\circ}\right)$, as large as the size of the visual displacement, and little aftereffect was observed $(-2.4 \mathrm{~mm})$. Conversely, in the contralateral shift condition, prism adaptation was enhanced (Fig. $3 c$ ) compared with the no-shift condition (Fig. $3 b$ ); in this case, the error during the exposure period decreased from $20 \mathrm{~mm}$ beyond the zero level and converged to $-3.9 \mathrm{~mm}$, and the estimated size of the aftereffect was $23 \mathrm{~mm}$, larger than the visual displacement.

The results were basically similar when the data obtained from six participants were pooled (Fig. $3 d-f, 12$ sessions). The plateau during the exposure period decreased in the following order: 12 $\mathrm{mm}$ in the ipsilateral shift condition, $4.1 \mathrm{~mm}$ in the no-shift condition, and $-0.96 \mathrm{~mm}$ in the contralateral shift condition. Conversely, the aftereffect increased in the following order: ipsilateral $(7.2 \mathrm{~mm})$, no-shift $(14 \mathrm{~mm})$, and contralateral shift (19 $\mathrm{mm}$ ) conditions. One-way repeated-measures ANOVA applied to the size of asymptotes estimated for each session showed that the main effect of the shift condition was significant $\left(F_{(2,22)}=21\right.$, $p<0.0001$ ). Post hoc tests (Ryan's test) showed that the mean asymptote was significantly larger in the ipsilateral shift condition $(10 \pm 2.0 \mathrm{~mm}$, mean \pm SEM; $p=0.0018)$ and significantly smaller in the contralateral shift condition $(-0.87 \pm 0.93 \mathrm{~mm}$, $p=0.0077)$ than in the no-shift condition $(4.2 \pm 0.81 \mathrm{~mm}$; Fig. $3 g)$. The main effect of the shift condition was significant for the size of the aftereffect as well $\left(F_{(2,22)}=8.4, p=0.0019\right)$. The mean aftereffect was significantly smaller in the ipsilateral shift condition $(7.5 \pm 2.4 \mathrm{~mm})$ than in the no-shift condition $(14 \pm 1.4 \mathrm{~mm}$, $p=0.029)$ or in the contralateral shift condition $(19 \pm 2.2 \mathrm{~mm}$, $p=0.00049$, Fig. $3 h$ ). Although the difference between the aftereffects in the contralateral shift condition and the no-shift con- 

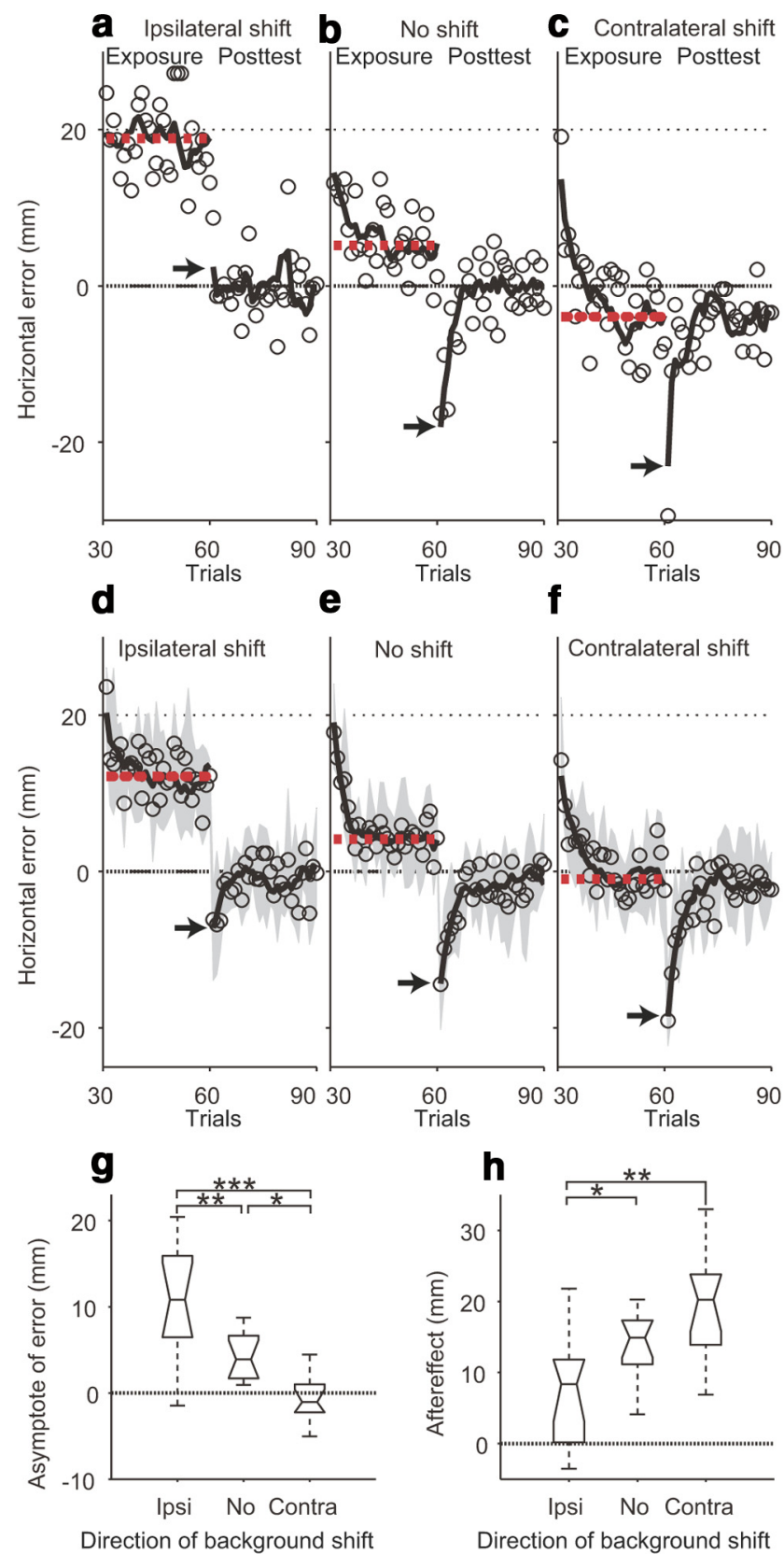

Figure 3. Effects of ipsilateral and contralateral shifts of the background (experiment 1). The data from an individual participant $(\boldsymbol{a}-\boldsymbol{c})$ and from all participants $(\boldsymbol{d}-\boldsymbol{f})$ are shown. Each circle represents the median of two $(\boldsymbol{a}-\boldsymbol{c})$ and $12(\boldsymbol{d}-\boldsymbol{f}, 2$ directions $\times 6$ participants) responses plotted against trial sequence (abscissa). Errors in the direction of prism displacement (right or left) are indicated as positive. The thick black lines indicate the model predictions using model equation in Materials and Methods. Note the difference in the asymptotes during the exposure period (red dotted lines) and in the aftereffects (black arrows) across three conditions: ipsilateral shift $(\boldsymbol{a}, \boldsymbol{d})$, no-shift $(\boldsymbol{b}, \boldsymbol{e})$, and contralateral shift $(\boldsymbol{c}, \boldsymbol{f})$. The gray shaded areas in $\boldsymbol{d}$ - $\boldsymbol{f}$ show the 25 th and 75 th percentiles. $\boldsymbol{g}, \boldsymbol{h}$, Distributions of the asymptotes during the exposure period $(\boldsymbol{g})$ and aftereffects during the posttest period ( $\boldsymbol{h})$ estimated for each session $(n=12)$. Each box plot shows the 10th, 25th, 50th, 75th, and 90th percentiles. Brackets with asterisks indicate significant differences $\left({ }^{*} p<0.05 ;{ }^{* *} p<0.01 ;{ }^{* * *} p<0.001\right)$ after corrections for multiple comparisons (Ryan's method after one-way repeated-measures ANOVA).

dition did not reach the level of significance, the results generally agreed with the predictions from our hypothesis: prism adaptation was attenuated in the ipsilateral shift condition and enhanced in the contralateral shift condition compared with the no-shift condition.

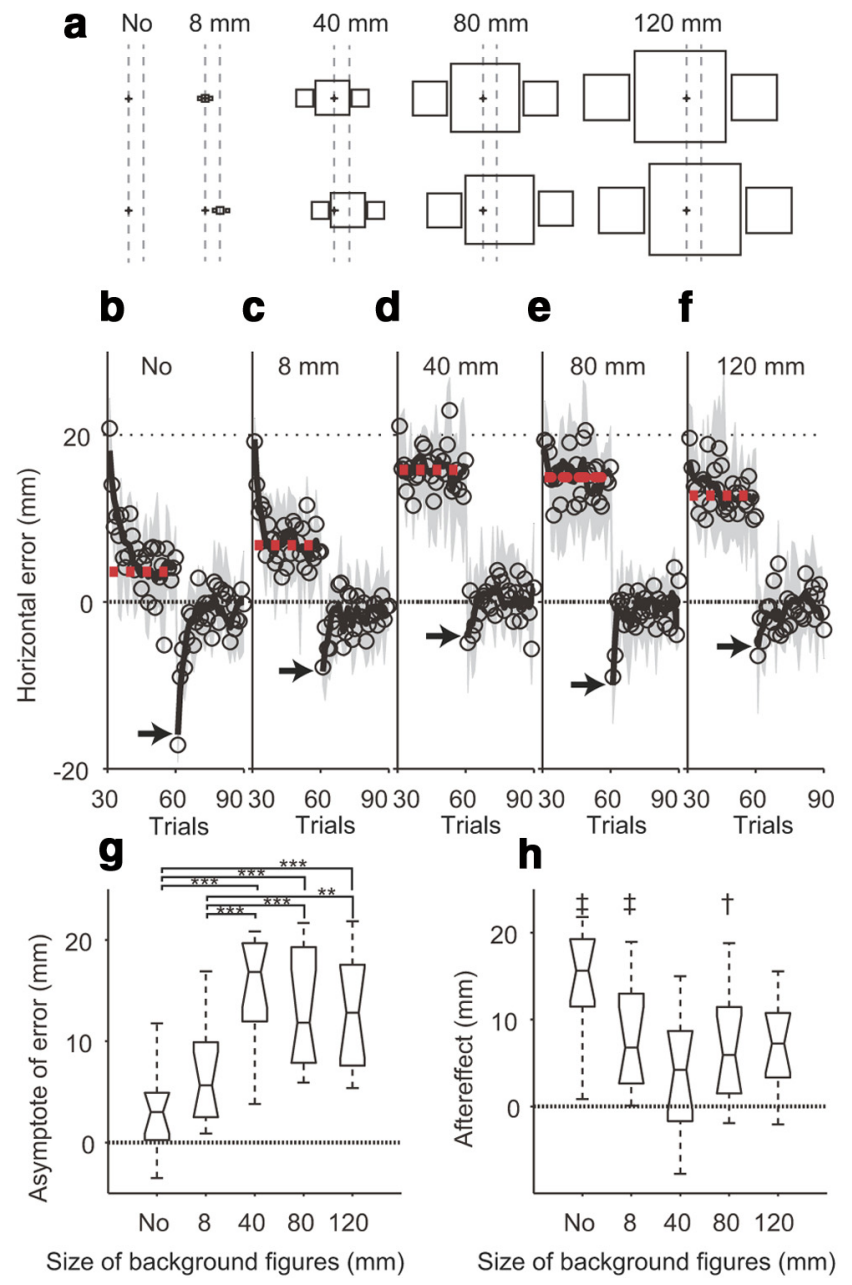

Figure 4. Effects of the size of the background (experiment 2) on cancelling. $\boldsymbol{a}$, Illustrations of background figures of different sizes. The size of the center square was 0 (no background), 8 , 40,80 , or $120 \mathrm{~mm}$. The top and bottom panels show the rightward shift of the background between the encoding (top) and decoding (bottom) phases. The vertical lines show the size of the shift $(20 \mathrm{~mm}) . \boldsymbol{b}-\boldsymbol{f}$, Prism adaptation with five different sizes of background. Note the maximal cancelling effects at $40 \mathrm{~mm}$. Each circle represents the median of 12 responses $(6$ participants $\times 2$ directions). $\boldsymbol{g}$, $\boldsymbol{h}$, Distributions of the asymptotes during the exposure period $(\boldsymbol{g})$ and aftereffects during the posttest period $(\boldsymbol{h})$. The daggers in $\boldsymbol{h}$ show that the mean aftereffect was significantly different from zero after correction for multiple comparisons $\left({ }^{\dagger} p<\right.$ $0.05 ;{ }^{\ddagger} p<0.01 ; t$ test, Bonferroni's correction). Other conventions are the same as in Figure 3 .

\section{Effects of the size of background (experiment 2)}

In experiment 1, the size of the center square was fixed at $80 \times 80$ $\mathrm{mm}$. In experiment 2 , we examined the effect of background size under the ipsilateral shift condition (Fig. $4 a$ ). The asymptote during the exposure period, estimated from the data pooled across six participants, changed with the size of the square in an inverted U-shaped manner with a peak at $40 \mathrm{~mm}$ (Fig. $4 b-f$ ), as follows: $3.6 \mathrm{~mm}$ (no figure), $6.8 \mathrm{~mm}$ (square width, $8 \mathrm{~mm}$ ), $16 \mathrm{~mm}$ (40 $\mathrm{mm}), 15 \mathrm{~mm}(80 \mathrm{~mm})$, and $13 \mathrm{~mm}(120 \mathrm{~mm})$. Accordingly, the size of the aftereffect changed in a $\mathrm{U}$-shaped manner with a minimum at $40 \mathrm{~mm}: 16 \mathrm{~mm}$ (no figure), $8.3 \mathrm{~mm}(8 \mathrm{~mm}), 4.3 \mathrm{~mm}(40$ $\mathrm{mm}), 10 \mathrm{~mm}(80 \mathrm{~mm})$, and $5.3 \mathrm{~mm}(120 \mathrm{~mm})$. One-way repeated-measures ANOVA applied to the size of the asymptotes estimated for each session ( $n=12$; Fig. $4 g$ ) showed that the main effect of the background size was significant $\left(F_{(4,44)}=19, p<\right.$ $0.0001)$. The main effect of the size was also significant for the size of the aftereffect $\left(F_{(4,44)}=2.6, p=0.046\right.$; Fig. 4h). Post hoc tests showed that the mean asymptote at $40 \mathrm{~mm}(15 \pm 1.7 \mathrm{~mm})$ was 
significantly larger than the mean with no background $(3.2 \pm 1.2$ $\mathrm{mm}, p<0.0001)$ and the mean at $8 \mathrm{~mm}(6.6 \pm 1.4 \mathrm{~mm}, p<$ $0.0001)$, although comparisons with the mean asymptote at 80 $\mathrm{mm}(14 \pm 1.7 \mathrm{~mm}, p=0.38)$ and $120 \mathrm{~mm}(13 \pm 1.7 \mathrm{~mm}, p=$ 0.24 ) did not reach the level of significance (Fig. $4 g$, brackets). Post hoc comparisons of the mean aftereffect did not reach the level of significance for any of the combinations (Fig. 4h). However, when the mean aftereffect was compared with zero, the aftereffect was significantly larger than zero at $8 \mathrm{~mm}(8.1 \pm 1.9$ $\mathrm{mm}, p=0.0014$; Bonferroni's correction for multiple comparison) and $80 \mathrm{~mm}(6.8 \pm 1.9 \mathrm{~mm}, p=0.0037)$ but not at $40 \mathrm{~mm}$ $(5.3 \pm 2.9 \mathrm{~mm}, p=0.098)$ or $120 \mathrm{~mm}(6.3 \pm 2.1 \mathrm{~mm}, p=0.011)$. These results show that the power of cancelling prism adaptation generally increased with the size of the square up to $40 \mathrm{~mm}$ but that it decreased or maintained a similar level with larger squares.

\section{Effects of the complexity of background (experiment 3)}

We then sought to determine which part of the background figures was essential for cancelling prism adaptation by using six background figures with different complexities (experiment 3; Fig. 5a): (1) no figure; (2) single line; (3) parallel lines; and an 80 mm square with 0 (4), 2 (5), and many smaller squares (6). As the complexity of the background figures increased, the asymptote during the exposure period generally increased, whereas the size of the aftereffect decreased (Fig. $5 b-g$ ). As a result, the cancelling effect was most prominent with the multiple-square background (Fig. 5g). One-way repeated-measures ANOVA showed that the main effect of the complexity of background figures was significant for the size of the asymptote $\left(F_{(5,55)}=8.7, p<0.0001\right.$; Fig. $5 h)$ as well as the size of the aftereffect $\left(F_{(5,55)}=4.2, p=0.0027\right.$; Fig. 5i). Post hoc tests (Ryan's test) showed that the mean asymptote became significantly larger than that in the no-figure condition $(4.1 \pm 0.89 \mathrm{~mm})$ with two parallel lines $(12 \pm 2.8 \mathrm{~mm}, p=$ $0.0013)$ and with more complex figures: a single square (12 \pm 2.3 $\mathrm{mm}, p=0.00035)$, triple squares $(13 \pm 3.1 \mathrm{~mm}, p=0.00030)$, and multiple squares $(14 \pm 2.1 \mathrm{~mm}, p<0.0001)$. The mean aftereffect became significantly smaller than that in the no-figure condition $(17 \pm 1.8 \mathrm{~mm})$ with a single square $(7.6 \pm 2.8 \mathrm{~mm}, p=$ $0.0019)$, triple squares $(8.0 \pm 3.1 \mathrm{~mm}, p=0.0027)$, and multiple squares $(5.9 \pm 2.4 \mathrm{~mm}, p=0.00026)$. Together, the results showed that the cancelling effect became significant with parallel lines and with more complicated figures, and the effect was most apparent with a square surrounded by many smaller squares (multiple squares).

\section{Necessity of the background during the encoding phase (experiment 4)}

We hypothesized that the initial target position is encoded in terms of the background figures before the blank and decoded after the blank, again in terms of the background. However, a single presentation of a large frame offset to the left or right of an observer's midline is known to induce a shift of the subjective straight ahead, or the egocentric coordinate, toward the offset of the frame (Roelofs effect; Roelofs, 1935; Bridgeman and Klassen, 1983; Bridgeman et al., 1997; Dassonville and Bala, 2004; Dassonville et al., 2004). Thus, it may be argued that the effect of cancelling prism adaptation was not attributable to allocentric encoding and decoding of the target position but simply resulted from encoding and decoding in the egocentric coordinate, which was shifted toward the offset of the background after the blank. To test this possibility, we examined whether prism adaptation could be cancelled when the target was presented without any background (thus encoded in the egocentric coordinate) during
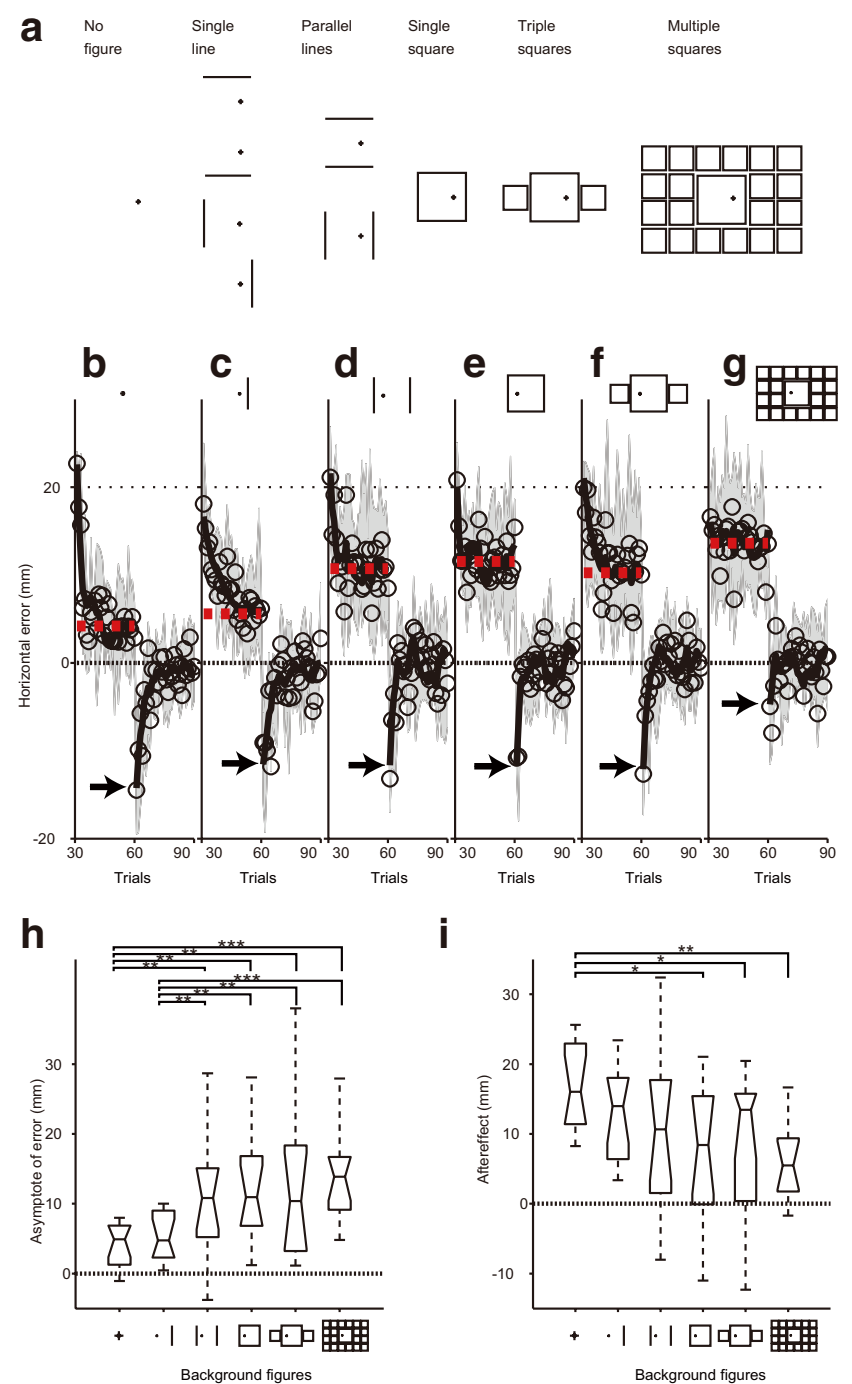

Figure 5. Effects of the complexity of the background on cancelling (experiment 3). a, Illustrations of background figures with different complexities: no background, a single line, parallel lines, a single square, triple squares, and multiple squares. The size of the center figure was fixed at $80 \mathrm{~mm} . \boldsymbol{b}-\boldsymbol{g}$, Prism adaptation with background figures of different complexities. Note the maximal cancelling effects with multiple squares. Each circle represents the median of 12 responses (12 participants $\times 1$ direction). $\boldsymbol{h}, \boldsymbol{i}$, Distributions of the asymptotes during the exposure period $(\boldsymbol{h})$ and aftereffects during the posttest period (i). Other conventions are the same as in Figure 3.

the encoding phase, and the background was then presented after the blank in a position shifted in the direction of visual displacement (Fig. 2b).

When the background was presented exclusively after the blank (Fig. $6 b$, post-presentation condition), prism adaptation was little affected. This finding was in marked contrast to the apparent cancellation (a large asymptote and a small aftereffect) observed when the background was presented before and after the blank with a shift in the ipsilateral direction (Fig. $6 a$, ipsilateral shift condition). One-way repeated-measures ANOVA and post hoc tests confirmed these observations: the mean asymptote in the post-presentation condition $(3.7 \pm 1.1 \mathrm{~mm})$ was not significantly different from the mean asymptote in the nobackground condition ( $3.8 \pm 0.70 \mathrm{~mm}, p=0.99)$, but it was significantly smaller than the mean asymptote in the ipsilateral shift condition $(13 \pm 2.9 \mathrm{~mm}, p=0.0011$; Fig. $6 d)$. The mean aftereffect in the post-presentation condition $(14 \pm 1.7 \mathrm{~mm})$ was 


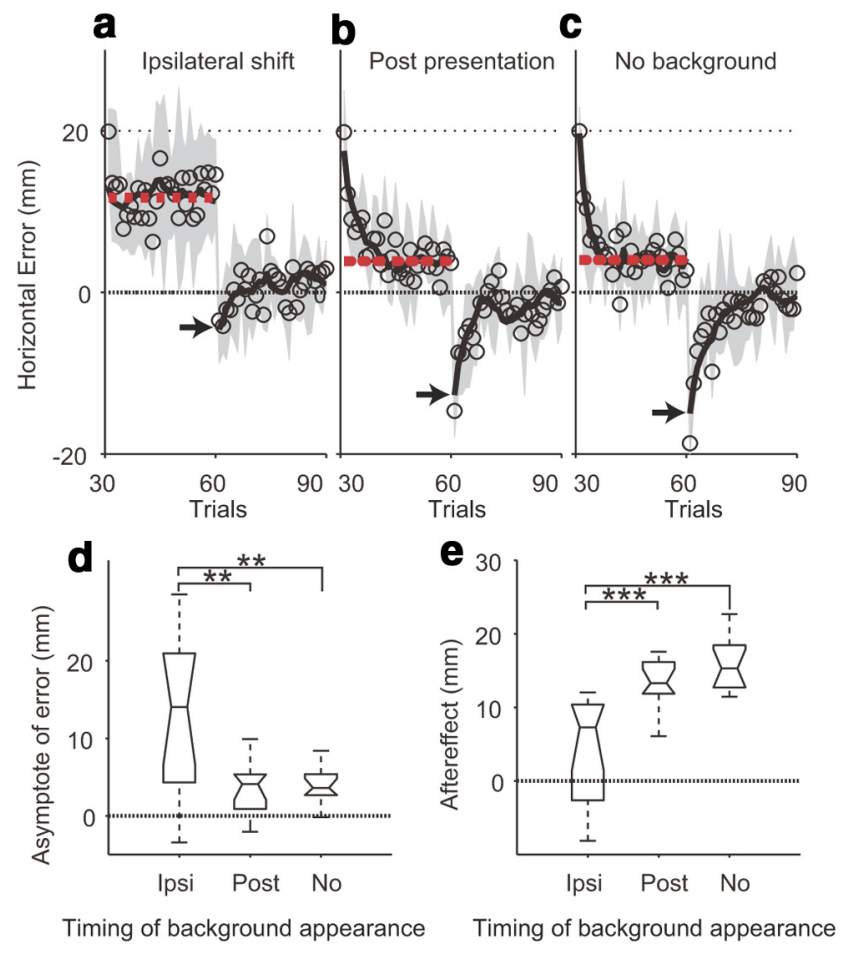

Figure 6. Necessity of the background during the encoding phase (experiment 4) for cancelling. $\boldsymbol{a}-\boldsymbol{c}$, Prism adaptation in the ipsilateral shift $(\boldsymbol{a})$, post-presentation $(\boldsymbol{b})$, and no background conditions. Note the contrast between the ipsilateral shift condition and the post-presentation condition. Each circle represents the median of 12 responses (6 participants $\times 2$ directions). $\boldsymbol{d}, \boldsymbol{e}$, Distributions of the asymptotes during the exposure period $(\boldsymbol{d})$ and aftereffects during the post-test period $(\boldsymbol{e})$. Other conventions are the same as in Figure 3.

as large as that in the no-background condition $(15 \pm 1.5 \mathrm{~mm}$, $p=0.51)$ but significantly larger than in the ipsilateral shift condition ( $4.2 \pm 2.1 \mathrm{~mm}, p=0.00020$; Fig. $6 e$ ). These results clearly show that presentation of the background was necessary not only during the decoding phase but also during the encoding phase to cancel prism adaptation; they exclude the possibility that the cancellation of prism adaptation was attributable to the shift of the egocentric coordinate resulting from the Roelofs effect.

\section{Discussion}

In the present study, we proposed a novel use of the allocentric memory of target position in an immediate reach. We hypothesized that the initial position of the target is encoded in terms of the background figures when we plan a reaching movement and that the initial position is decoded in terms of the background to evaluate the motor error when the movement is completed. Assuming that this hypothesis is correct, we would be able to control the "motor error" in prism adaptation by shifting the background in flight of the reach while keeping the actual target position unchanged. Our results supported the hypothesis: we were able to cancel or enhance prism adaptation by shifting a background in flight of the reach in the direction of visual displacement (cancellation) or in the opposite direction (enhancement).

\section{Was it really allocentric?}

The results supported our hypothesis that the initial target position is encoded and decoded in terms of allocentric coordinates. However, it may still be argued that the error was encoded in the egocentric coordinate and decoded again in the egocentric coordinate that was shifted toward the offset of the background fig- ures. This argument is reasonable because it is well known that the subjective midline, which forms the basis of the egocentric coordinate, is shifted toward the center of a large frame presented with an offset to the right or to the left (Roelofs effect; Roelofs, 1935; Bridgeman and Klassen, 1983). Accordingly, a target position is mislocalized in the opposite direction in such a way that a target presented in the actual midline is judged as localized to the left of the midline when a frame is presented with an offset to the right and vice versa (induced Roelofs effect; Bridgeman et al., 1997). The induced Roelofs effect is now generally accepted to result from the shift of the egocentric coordinate toward the offset of the large frame (Dassonville and Bala, 2004; Dassonville et al., 2004).

If the cancellation and enhancement observed in our experiments was caused by the shift of the egocentric coordinate resulting from the Roelofs effect, cancellation of prism adaptation should be observed simply by presenting a frame with an offset after the reaching was completed, without presenting any background at the time when the movement was planned (postpresentation condition). We tested this in experiment 4 , and cancellation was not observed in the post-presentation condition. The results excluded the possibility that the cancellation was caused by the shift of egocentric coordinate resulting from the Roelofs effect.

\section{Relationship with nonretinotopic exogenous attention}

A recent study reported that reflexive, stimulus-driven exogenous attention operates not only in the retinotopic coordinates but also in allocentric coordinates (Boi et al., 2011). In the study, a cue was briefly presented in the first frame on a background that consisted of three squares, and then in the second frame, the background was shifted to the right or to the left. Exogenous attention was shown to be elevated in the second frame at a cued location in terms of the background as well as at a cued location in terms of the retinotopic coordinate. However, the exogenous attentional capture was transient. The effect peaked at $170 \mathrm{~ms}$ and disappeared before 300 ms after the cue onset. Thus, the nonretinotopic exogenous attention should have disappeared during the reaching movement, because the summation of the reaction time (150-300 $\mathrm{ms})$ and the movement duration ( $\sim 250 \mathrm{~ms}$ on average) was longer than $300 \mathrm{~ms}$. However, it is still possible that encoding of the initial target position may share some resources with the nonretinotopic exogenous attention.

\section{Merits of using the background coordinate}

To generate motor commands that achieve an appropriate reaching movement toward a target, it is necessary to encode the target position in terms of an egocentric coordinate (Kawato et al., 1987; Soechting and Flanders, 1989; McIntyre et al., 1997; Henriques et al., 1998; Vetter et al., 1999; Blohm and Crawford, 2007; Chen et al., 2011; Tanaka and Sejnowski, 2013). The question then arises as to why the brain takes the trouble of using the allocentric coordinate instead of the egocentric coordinate for encoding and decoding the initial target position.

One possible reason for this can be suggested based on the lifespans of the allocentric and egocentric memories. Previous studies reported that the allocentric memory of target position survives longer than the egocentric memory in a delayed reaching task (McIntyre et al., 1998). It is thus possible that the brain had to rely on the allocentric memory because the egocentric memory faded during the $300 \mathrm{~ms}$ blank period. However, this is unlikely because the merit of allocentric memory in delayed reaching be- 
came significant only when the delay period was longer than $1 \mathrm{~s}$ (Sheth and Shimojo, 2004; Obhi and Goodale, 2005), much longer than the duration of the blank period in the present study (300 ms).

We suggest another clear advantage of using the allocentric memory for memorizing the initial target position. Because participants generally make a saccade during the blank period, it is useless to rely on the retinotopic coordinate for memorizing the initial target position. The brain has to rely on the cranial coordinate, but the resolution in the cranial coordinate depends on the error in evaluating the eye position. In natural situations, the head also moves during the reach. Thus, the initial target position should be decoded in terms of the body, and the errors in estimating the eye and head positions additively blur the memory of the initial target position. Conversely, the background is generally stable in natural situations. Thus, the allocentric coordinate offers a reliable frame of reference that is immune to the movements of the body parts. This explains why the brain relies on the allocentric coordinate for evaluating the initial target position and the motor error.

\section{Optimal size and complexity of a background}

The smallest figure $(8 \mathrm{~mm})$ in experiment 2 , which was as small as the target itself, did not yield any significant cancelling effect, nor did the effect of a single line $80 \mathrm{~mm}$ in length in experiment 3 reach a level of significance. However, two lines achieved a level of significance. These results generally suggest that the target should be "surrounded" by salient landmarks at the time of encoding to form an effective background coordinate.

With respect to the size of the center square that surrounded the target (experiment 2), the cancelling effect was optimal when the center square was $40 \mathrm{~mm}$ compared with when the center square was 80 or $120 \mathrm{~mm}$. The optimal size can be explained in terms of the minimal distance between the target and the square at the time of encoding. Lemay et al. (2004) reported that the error in a delayed reaching task with a background square became smaller when the target was located closer to the side of the square. In the present study, a target was presented at a random location within a circle (20 $\mathrm{mm}$ radius) with its center at the center of the middle square. Thus, the minimal distance ranged from 0 to $20 \mathrm{~mm}$ when the size of the square was $40 \mathrm{~mm}$; this was much smaller than the distances with larger squares $(20-40 \mathrm{~mm}$ at width of $80 \mathrm{~mm}$ and $40-60 \mathrm{~mm}$ at width of $120 \mathrm{~mm})$. Similar results were reported by Krigolson et al. (2007).

The optimal size of the background can also be explained in terms of the saliency of the change attributable to the shift of the background by $20 \mathrm{~mm}$. As mentioned, the target was presented at a random location within a circle (20 mm radius). Thus, the target was located outside the center square with a probability of 0.5 when the center square was $40 \mathrm{~mm}$ in width. In contrast, the target remained within the center square when the square was 80 or $120 \mathrm{~mm}$ wide. The "jump" over the vertical side of the square would serve as a strong indication that a target has moved out of the frame. In this case, the brain would have directly depended on the allocentric memory of the initial target position within the square to evaluate the motor error.

Finally, it is worth noting that the cancelling effect with the 80 $\mathrm{mm}$ square was much enhanced by adding many smaller squares. Because the distribution of the distance between the target and each side of the square was unchanged, the results indicate that the "mass" of the figures represents another important factor in whether the figures are regarded as a background.
Natural scenes are full of details and salient features that surround a target to be reached. It is thus reasonable to expect that the background coordinate is actually used in our daily lives for evaluating the error of our reaching movements by presenting the initial target position. The remaining question is how the initial target position is represented in terms of the background in the brain. One extreme possibility is that our brain takes a snapshot of the landscape and maps the initial target position on the snapshot. A new snapshot after a blank is overlaid to the old one on the basis of pixelwise similarity, so that a heavy background would dominate over a single target. Another extreme possibility is that the landscape is parsed into a number of independent objects, each of which and the target itself may be represented even in the egocentric coordinate. Then, the initial target position is encoded as a combination of vectors drawn from each object to the target. Assuming that this hypothesis is true, allocentric process could be performed by neurons that encode object positions in egocentric coordinates and that the brain may not distinguish between target and background, per se. Either theory does not contradict with the results at present. Whether and how the "background coordinate" is represented in the brain merits additional investigation.

\section{References}

Blohm G, Crawford JD (2007) Computations for geometrically accurate visually guided reaching in 3-D space. J Vis 7:4 1-22. CrossRef Medline

Boi M, Vergeer M, Ogmen H, Herzog MH (2011) Nonretinotopic exogenous attention. Curr Biol 21:1732-1737. CrossRef Medline

Bridgeman B, Klassen H (1983) On the origin of stroboscopic induced motion. Percept Psychophys 34:149-154. CrossRef Medline

Bridgeman B, Peery S, Anand S (1997) Interaction of cognitive and sensorimotor maps of visual space. Percept Psychophys 59:456-469. CrossRef Medline

Burgess N, Spiers HJ, Paleologou E (2004) Orientational manoeuvres in the dark: dissociating allocentric and egocentric influences on spatial memory. Cognition 94:149-166. CrossRef Medline

Byrne PA, Crawford JD (2010) Cue reliability and a landmark stability heuristic determine relative weighting between egocentric and allocentric visual information in memory-guided reach. J Neurophysiol 103:30543069. CrossRef Medline

Byrne PA, Cappadocia DC, Crawford JD (2010) Interactions between gazecentered and allocentric representations of reach target location in the presence of spatial updating. Vision Res 50:2661-2670. CrossRef Medline

Carrozzo M, Stratta F, McIntyre J, Lacquaniti F (2002) Cognitive allocentric representations of visual space shape pointing errors. Exp Brain Res 147: 426-436. CrossRef Medline

Chen Y, Byrne P, Crawford JD (2011) Time course of allocentric decay, egocentric decay, and allocentric-to-egocentric conversion in memoryguided reach. Neuropsychologia 49:49-60. CrossRef Medline

Dassonville P, Bala JK (2004) Perception, action, and Roelofs effect: a mere illusion of dissociation. PLoS Biol 2:e364. CrossRef Medline

Dassonville P, Bridgeman B, Kaur Bala J, Thiem P, Sampanes A (2004) The induced Roelofs effect: two visual systems or the shift of a single reference frame? Vision Res 44:603-611. CrossRef Medline

Day BL, Lyon IN (2000) Voluntary modification of automatic arm movements evoked by motion of a visual target. Exp Brain Res 130:159-168. CrossRef Medline

Day RW, Quinn GP (1989) Comparisons of treatments after an analysis of variance in ecology. Ecol Monogr 59:433-463. CrossRef

Henriques DY, Klier EM, Smith MA, Lowy D, Crawford JD (1998) Gazecentered remapping of remembered visual space in an open-loop pointing task. J Neurosci 18:1583-1594. Medline

Kawato M, Furukawa K, Suzuki R (1987) A hierarchical neural-network model for control and learning of voluntary movement. Biol Cybern 57: 169-185. CrossRef Medline

Kitazawa S, Yin PB (2002) Prism adaptation with delayed visual error signals in the monkey. Exp Brain Res 144:258-261. CrossRef Medline

Kitazawa S, Kohno T, Uka T (1995) Effects of delayed visual information on 
the rate and amount of prism adaptation in the human. J Neurosci 15: 7644-7652. Medline

Krigolson O, Heath M (2004) Background visual cues and memory-guided reaching. Hum Mov Sci 23:861-877. CrossRef Medline

Krigolson O, Clark N, Heath M, Binsted G (2007) The proximity of visual landmarks impacts reaching performance. Spat Vis 20:317-336. CrossRef Medline

Lemay M, Bertram CP, Stelmach GE (2004) Pointing to an allocentric and egocentric remembered target. Motor Control 8:16-32. Medline

McIntyre J, Stratta F, Lacquaniti F (1997) Viewer-centered frame of reference for pointing to memorized targets in three-dimensional space. J Neurophysiol 78:1601-1618. Medline

McIntyre J, Stratta F, Lacquaniti F (1998) Short-term memory for reaching to visual targets: psychophysical evidence for body-centered reference frames. J Neurosci 18:8423-8435. Medline

Obhi SS, Goodale MA (2005) The effects of landmarks on the performance of delayed and real-time pointing movements. Exp Brain Res 167:335-344. CrossRef Medline
Oldfield RC (1971) The assessment and analysis of handedness: the Edinburgh inventory. Neuropsychologia 9:97-113. CrossRef Medline

Roelofs C (1935) Optische Localisation. Arch Augenheilkunde 109: 395-415.

Sheth BR, Shimojo S (2004) Extrinsic cues suppress the encoding of intrinsic cues. J Cogn Neurosci 16:339-350. CrossRef Medline

Soechting JF, Flanders M (1989) Sensorimotor representations for pointing to targets in three-dimensional space. J Neurophysiol 62: 582-594. Medline

Tanaka H, Sejnowski TJ (2013) Computing reaching dynamics in motor cortex using Cartesian spatial coordinates. J Neurophysiol 109:11821201. CrossRef Medline

Vetter P, Goodbody SJ, Wolpert DM (1999) Evidence for an eye-centered spherical representation of the visuomotor map. J Neurophysiol 81:935939. Medline 